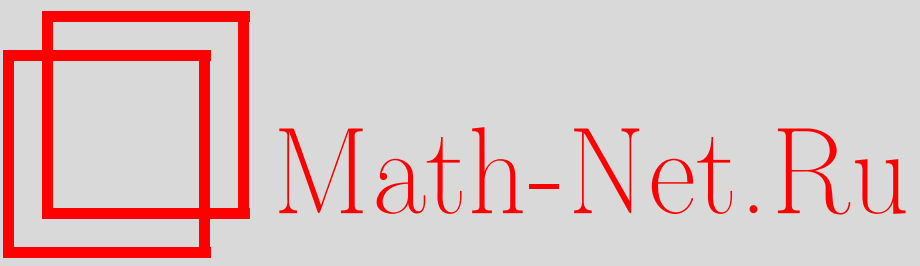

Л. Г. Рыбников, Слабо коммутативные однородные пространства с редуктивным стабилизатором, УМН, 2004, том 59, выпуск 4, 199-200

DOI: https://doi.org/10.4213/rm766

Использование Общероссийского математического портала Math-Net.Ru подразумевает, что вы прочитали и согласны с пользовательским соглашением

http://www.mathnet.ru/rus/agreement

Параметры загрузки:

IP : 54.196 .121 .252

26 апреля 2023 г., 13:27:26 


\section{СЛАБО КОММУТАТИВНЫЕ ОДНОРОДНЫЕ ПРОСТРАНСТВА С РЕДУКТИВНЫМ СТАБИЛИЗАТОРОМ}

\section{Л. Г. РыБников}

1. Пусть $G$ - вещественная алгебраическая группа, $\mathfrak{g}=\operatorname{Lie} G, H$ - редуктивная подгруппа в $G, \mathfrak{h}=$ Lie $H$. На алгебре $D(X)$ дифференциальных операторов на однородном пространстве $X=G / H$ имеется естественная фильтрация по порядку дифференциального оператора. Коммутатор в алгебре $D(X)$ задает на ассоциированной градуированной алгебре $P(X)=\operatorname{gr} D(X)$ скобку Пуассона.

Пусть $D(X)^{G}$ и $P(X)^{G}$ - подалгебры $G$-инвариантов в $D(X)$ и $P(X)$ соответственно. Известно [1], что $D(X)^{G} \simeq(U(\mathfrak{g}) / U(\mathfrak{g}) \mathfrak{h})^{H}$ как фильтрованные ассоциативные алгебры. На уровне пуассоновых алгебр имеется изоморфизм $P(X)^{G} \simeq(S(\mathfrak{g}) / S(\mathfrak{g}) \mathfrak{h})^{H}$. Из этого описания, в силу редуктивности группы $H$, следует, что gr $D(X)^{G}=P(X)^{G}$.

Однородное пространство $X$ называется коммутативным, если алгебра $D(X)^{G}$ коммутативна, и слабо коммутативным, если алгебра Пуассона $P(X)^{G}$ коммутативна. Так как $\operatorname{gr} D(X)^{G}=P(X)^{G}$, то из коммутативности однородного пространства следует его слабая коммутативность. Мы докажем следующую теорему о структуре слабо коммутативных однородных пространств.

Теорема 1. Для любого слабо коммутативного однородного пространства $X=$ $G / H$ существует такое разложение Леви $G=L \curlywedge N$, что

1) $L$ - редуктивная подгруппа Ли, содержащая $H$;

2) $S(\mathfrak{n})^{L}=S(\mathfrak{n})^{H}$.

В [2] утверждение теоремы 1 доказано для римановых однородных пространств (т.е. для пространств $G / H$ с компактной группой $H$ ). В этом случае степень нильпотентности группь $N$ не превосходит 2 . Мы покажем, что в общем случае группа $N$ может иметь любую степень нильпотентности.

В [3] доказано, что любое слабо коммутативное риманово однородное пространство коммутативно. Мы докажем следующую теорему о коммутативности некоторых (вообще говоря, не римановых) слабо коммутативных однородных пространств.

Теорема 2. Пусть $X=G / H$ - слабо коммутативное однородное пространство, причем

1) унипотентный радикал $N$ группь $G$ есть $(2 n-1)$-мерная группа Гейзенберга;

2) группа $H$ действует тривиально на (одномерном) чентре группы $N$.

Тогда пространство $X$ коммутативно.

Автор благодарен Э.Б. Винбергу за постановку задачи и постоянное внимание к работе.

2. Докажем теорему 1. Так как группа $H$ редуктивна, то можно выбрать $L$ так, что $H \subset$ $L$. Так как группа $L$ редуктивна, то на пространстве $\mathfrak{l}$ имеется невырожденная $L$-инвариантная квадратичная форма. Так как группа $H$ редуктивна, то ограничение этой формы на подпространство $\mathfrak{h} \in \mathfrak{l}$ невырождено. Отсюда следует, что на пространстве $(\mathfrak{l} / \mathfrak{h})^{*}$ имеется невырожденная $H$-инвариантная квадратичная форма. Эта форма определяет многочлен второй степени $\Phi \in\left(S^{2}(\mathfrak{l} / \mathfrak{h})\right)^{H} \subset(S(\mathfrak{g} / \mathfrak{h}))^{H}$.

Для любого многочлена $\Psi \in S(\mathfrak{n})^{H} \subset(S(\mathfrak{g} / \mathfrak{h}))^{H}$ условие $\{\Phi, \Psi\}=0$ равносильно условию $\{x, \Psi\}=0$ для любого $x \in \mathfrak{l}$ или, что то же самое, $\Psi \in S(\mathfrak{n})^{L}$. Так как пространство $X$ слабо коммутативно, то для любого $\Psi \in S(\mathfrak{n})^{H} \subset(S(\mathfrak{g} / \mathfrak{h}))^{H}$ выполнено условие $\{\Phi, \Psi\}=0$. Следовательно, $S(\mathfrak{n})^{L}=S(\mathfrak{n})^{H}$.

ЗАмечАниЕ 1 . На степень нилпотентности группы $N$ нет никаких ограничений. Пусть $V$ и $W$-векторные пространства над $\mathbb{R}$, причем $\operatorname{dim} V=n+1, \operatorname{dim} W=n$. Рассмотрим $n$-ступенно 
нильпотентную группу $N=(\exp \operatorname{Id} \otimes \mathbb{R} J)<(V \otimes W)$, где $J \in \operatorname{End}(W)$ - нильпотентный оператор ранга $n-1$. Однородное пространство $X=(S L(V) \wedge N) / S L(V)$ коммутативно: алгебра $D(X)^{G}=U(\mathfrak{n})^{S L(V)}$ есть алгебра многочленов от одного переменного.

3. Теперь докажем теорему 2 . Выберем разложение Леви $G=L \wedge N$ как в теореме 1 . Пусть $\mathfrak{z} \subset \mathfrak{n}$-центр алгебры Ли $\mathfrak{n}=\operatorname{Lie} N$ и $\mathfrak{a} \subset \mathfrak{n}$-некоторое $L$-инвариантное дополнительное подпространство. Пусть $z$-ненулевой элемент в $\mathfrak{z}$. Из теоремы 1 следует, что $z \in \mathfrak{n}^{G}$. Это означает, что на пространстве $\mathfrak{a}$ имеется невырожденная $L$-инвариантная кососимметрическая форма $\omega$ такая, что для $v, w \in \mathfrak{a}$ имеем $[v, w]=\omega(v, w) z$.

Так как $z \in \mathfrak{n}^{G}$, то имеется естественное вложение алгебры $\mathbb{R}[z]$ в центр алгебры $D(X)^{G} \simeq$ $(U(\mathfrak{g}) / U(\mathfrak{g}) \mathfrak{h})^{H}$. Это означает, что алгебру $D(X)^{G}$ можно рассматривать как алгебру регулярных сечений расслоения над $\operatorname{Spec} \mathbb{R}[z]=\mathfrak{z}^{*}$, слой которого над точкой $z=t$ есть алгебра $D(X)_{t}^{G}=D(X)^{G} / D(X)^{G}(z-t)$. Аналогично, алгебру Пуассона $P(X)^{G}$ можно рассматривать как алгебру регулярных сечений расслоения над $\operatorname{Spec} \mathbb{R}[z]=\mathfrak{z}^{*}$, слой которого над точкой $z=t$ есть алгебра $P(X)_{t}^{G}=P(X)^{G} / P(X)^{G}(z-t)$.

Пусть $W(\mathfrak{a})$ - алгебра Вейля с пространством образующих $\mathfrak{a}$ и определяющими соотношениями $v w-w v=\omega(v, w)$ для любых $v, w \in \mathfrak{a}$. На алгебре $W(\mathfrak{a})$ имеется фильтрация по степени выражения через образующие, причем ассоциированная градуированная (пуассонова) алгебра изоморфна $S(\mathfrak{a})$. Группа $L$ действует на пространстве a преобразованиями, сохраняющими форму $\omega$. Определим "полупрямое тензорное произведение" $U(\mathfrak{l}) \bigotimes W(\mathfrak{a}):$ это алгебра, порожденная $U(\mathfrak{l})$ и $W(\mathfrak{a})$ с определяющими соотношениями $x a-a x=x(a) \forall x \in \mathfrak{l}, a \in \mathfrak{a}$. Рассмотрим подалгебру $H$-инвариантов в алгебре $U(\mathfrak{g}) \bigotimes W(\mathfrak{a})$. Очевидно, что $(U(\mathfrak{l}) \mathfrak{h} \bigotimes W(\mathfrak{a}))^{H}$ - двусторонний идеал в $U(\mathfrak{l}) \bigotimes W(\mathfrak{a}))^{H}$. Обозначим соответствующую факторалгебру через $B(L, H, \mathfrak{a})$. Ассоциированная алгебра Пуассона изоморфин $(S(\mathfrak{g} / \mathfrak{h}) \otimes S(\mathfrak{a}))^{H}=\mathbb{R}\left[(\mathfrak{l} / \mathfrak{h})^{*} \times \mathfrak{a}^{*}\right]^{H}$

ЛЕмма 1 [3]. При $t \neq 0$ имеет место изоморфизм ассоциативньх алгебр $D(X)_{t}^{G} \simeq$ $B(L, H, \mathfrak{a})$, а также изоморфизм алгебр Пуассона $P(X)_{t}^{G} \simeq S((\mathfrak{l} / \mathfrak{h}) \times \mathfrak{a})^{H}$.

Так как пространство $X$ слабо коммутативно, то алгебра Пуассона $P(X)_{t}^{G} \simeq S((\mathfrak{l} / \mathfrak{h}) \times \mathfrak{a})^{H}$ коммутативна. На пространстве $(\mathfrak{l} / \mathfrak{h})^{*} \times \mathfrak{a}^{*}$ имеется невырожденная $H$-инвариантная билинейная форма (симметрическая на $(\mathfrak{l} / \mathfrak{h}){ }^{*}$ и кососимметрическая на $\left.\mathfrak{a}^{*}\right)$, поэтому представление редук тивной группы $H$ в пространстве $(\mathfrak{l} / \mathfrak{h})^{*} \times \mathfrak{a}^{*}$ самосопряжено. Отсюда следует, что $H$-орбиты общего положения в $(\mathfrak{l} / \mathfrak{h})^{*} \times \mathfrak{a}^{*}$ разделяются полиномиальными инвариантами.

ЛЕмма 2 [3]. Пусть L - редуктивная группа, действующая симплектическими преобразованиями на пространстве а с невьрожсденой 2-формой $\omega, H$ - подгруппа в $L$. Пусть $Н$-орбиты общего положения в $(\mathfrak{l} / \mathfrak{h})^{*} \times \mathfrak{a}^{*}$ разделяются полиномиальными инвариантами. Тогда из коммутативности алгебры Пуассона $S((\mathfrak{l} / \mathfrak{h}) \times \mathfrak{a})^{H}$ следует коммутативность ассоциативной алгебры $B(L, H, \mathfrak{a})$.

Доказательство основано на построении гомоморфизма центра универсалнной обертывающей алгебры $U(\mathfrak{l} \oplus \mathfrak{s p}(\mathfrak{a}, \omega))$ в алгебру $B(L, H, \mathfrak{a})$, образ которого является "достаточно большой” коммутативной подалгеброй в $B(L, H, \mathfrak{a})$ в том смысле, что алгебра $B(L, H, \mathfrak{a})$ является ее алгебраическим расширением.

Из леммы 2 следует коммутативность алгебры $D(X)_{t}^{G}$ при всех $t \neq 0$, а значит, и коммутативность алгебры $D(X)^{G}$.

\section{СПИСОК ЛИТЕРАТУРЫ}

[1] S. Helgason // Acta Math. 1959. V. 102. P. 239-299. [2] Э. Б. Винберг // УМН. 2001. Т. 56. № 1. С. 3-62. [3] Л. Г. Рыбников // Функц. анализ и его прил. 2003. Т. 37. № 2. С. 41-51.

Московский государственный университет им. М.В. Ломоносова
Принято редколлегией 24.05.2004 